\title{
Superior Specimen and Diagnostic Accuracy with Endoscopic Ultrasound-Guided Liver Biopsies Using 19-Gauge versus 22-Gauge Core Needles
}

\author{
Rucha M. Shah ${ }^{1}$, Jason Schmidt ${ }^{2}$, Elizabeth John', Sheila Rastegari ${ }^{3}$, Priyanka Acharya ${ }^{4}$ and Prashant Kedia ${ }^{1,5}$ \\ ${ }^{1}$ Department of Gastroenterology, Methodist Dallas Medical Center, Dallas, TX, ${ }^{2}$ Department of Pathology, Methodist Dallas Medical \\ Center, Dallas, TX, ${ }^{3}$ Department of Internal Medicine, Methodist Dallas Medical Center, Dallas, TX, ${ }^{4}$ Methodist Health System Clinical \\ Research Institute, Methodist Dallas Medical Center, Dallas, TX, ${ }^{5}$ Digestive Health Associates of Texas, Dallas, TX, USA
}

Background/Aims: Endoscopic ultrasound-guided liver biopsy (EUS-LB) is an effective and safe method of procuring liver tissue. The aims of this study were to assess and compare the outcomes and tissue adequacy of a single-pass, single-actuation, wet suction technique between $19 \mathrm{G}$ and $22 \mathrm{G}$ needles in patients undergoing EUS-LB.

Methods: We performed a prospective case series study of 20 patients undergoing EUS-LB at a single center between September 2017 and April 2020. The primary objective was to evaluate differences in sample adequacy via a single actuation wet suction technique between a $19 \mathrm{G}$ core needle and a $22 \mathrm{G}$ core needle. Adequacy was gauged by cumulative core biopsy length and the number of portal tracts visualized.

Results: The $19 \mathrm{G}$ needle provided a longer core length $(2.5 \mathrm{~cm}$ vs. $1.2 \mathrm{~cm}, p<0.0001)$, more complete portal tracts (5.8 vs. 1.7 , $p<0.0001)$, more total tracts ( 8.8 vs. $3, p<0.0001)$, and a longer, intact, fragment length $(0.75 \mathrm{~cm}$ vs. $0.32 \mathrm{~cm}, p<0.0006)$. The $19 \mathrm{G}$ needle was superior in providing adequate $(60 \%$ vs. $5 \%, p<0.001)$ and diagnostic pathologic samples $(85 \%$ vs. $10 \%, p<0.001)$.

Conclusions: A single-pass, single-actuation, wet suction technique using a $19 \mathrm{G}$ needle is superior to that using a $22 \mathrm{G}$ needle for tissue acquisition and sample adequacy in EUS-LB. Clin Endosc 2021;54:739-744

Key Words: 19 G FNB; Endoscopic ultrasound-guided liver biopsy; Liver biopsy

\section{INTRODUCTION}

The use of noninvasive biomarkers and imaging studies such as transient elastography has increased; however, liver biopsy continues to be a cornerstone in the diagnosis, staging, and management of patients with hepatic disease. ${ }^{1}$ Traditionally, liver biopsies have been performed via the transjugular or percutaneous routes. ${ }^{2}$ While these methods have been shown

Received: August 6, 2020 Revised: October 2, 2020

Accepted: October 6, 2020

Correspondence: Prashant Kedia

Digestive Health Associates of Texas, Pav. II, 221 W Colorado Blvd Ste. 630, Dallas, TX 75208, USA

Tel: +1-214-941-6891, Fax: +1-214-943-5871, E-mail: Kediaps@gmail.com ORCID: https://orcid.org/0000-0001-6472-0902

(c) This is an Open Access article distributed under the terms of the Creative Commons Attribution Non-Commercial License (http://creativecommons.org/ licenses/by-nc/3.0) which permits unrestricted non-commercial use, distribution, and reproduction in any medium, provided the original work is properly cited. to be effective and safe, they have limitations. A percutaneous liver biopsy may be anxiety-provoking, painful, limited by a large body habitus, and cannot provide bilobar sampling. Transjugular liver biopsies expose the patient to radiation and potential complications such as hematomas and pneumothoraces from central venous access. In addition, neither route allows for real-time visualization of the liver parenchyma during needle insertion to avoid blood vessels and other major structures. Endoscopic ultrasound-guided liver biopsy (EUSLB) sampling is emerging as an alternative to the aforementioned approaches, which offers several advantages without many of their limitations. ${ }^{1-4}$ A recent meta-analysis showed EUS-LB provides a histological diagnosis in over $93 \%$ of cases with an adverse event rate of $2.3 \%{ }^{5}$ Despite the growing and encouraging literature on EUS-LB, standardized practice has not been achieved in terms of approach, needle type or gauge, acquisition techniques, and tissue handling. Multiple studies 
have shown success with both 19 and 22 G needles; however, the number of needle actuations into the liver parenchyma was high, ranging from 9 to $20 .^{6-8}$ Theoretically, the risk of complications increases with the number of needle actuations. Thus, limiting this number while still procuring an adequate specimen is ideal. Nieto et al. described success with a single-pass, single-actuation wet suction technique using a $19 \mathrm{G}$ needle, where only two total needle actuations provided highly adequate specimens with a low adverse event rate of $1.8 \%{ }^{9}$ We chose to use the wet suction technique as presently it is the only technique in the literature published to date that requires only a single pass and single actuation of the EUS needle to procure an adequate liver biopsy specimen. The application of the single actuation wet suction technique has not been studied with different needle gauges. Therefore, the goal of this study was to assess the outcomes and tissue adequacy of performing a single pass, single actuation, and wet suction technique using specific $19 \mathrm{G}$ and $22 \mathrm{G}$ needles in patients, to help identify and standardize an EUS-LB technique that obtains adequate specimens and concurrently minimizes risk to the patient.

\section{MATERIALS AND METHODS}

This was a prospective case series of patients undergoing EUS-LB at a single center between September 2017 and April 2020. Patients were enrolled into the study if they were 18 years of age or older, with abnormal results for liver function tests of unclear etiology, with abnormal liver imaging, or needed staging for fibrosis, and were willing to undergo an EUSLB for evaluation. They were excluded if they were younger than 18 years of age or did not want to undergo an EUS-LB. The study protocol was approved by the Institutional Review Board, protocol number 045.GID.2017.D.

Our primary objective was to evaluate differences in liver biopsy sample adequacy obtained via a single actuation wet suction technique using a $22 \mathrm{G}$ core needle and a $19 \mathrm{G}$ core needle from the transgastric position into the left lobe of the liver. All EUS procedures were performed by a single high-volume endosonographer (PK). The indication for liver biopsy in all patients was to investigate elevated liver function tests, abnormal liver imaging, evaluation of fibrosis, or evaluation for acute cellular rejection after orthotopic liver transplant, as noted in Table 1. Adequacy was gauged by the cumulative length of the core biopsy and the number of portal tracts visualized by a single gastrointestinal pathologist, JS. In accordance with the American Association for the Study of Liver Disease, our specimen adequacy was defined as a specimen containing $\geq 11$ portal tracts or measuring $\geq 20 \mathrm{~mm}$ cumulative core length. ${ }^{10}$ Secondary objectives included evaluating the difference in overall diagnostic yield, adverse events, number of tissue fragments, and maximum fragment length between the two needle specimens. A specimen was deemed diagnostic when the pathologist was able to establish a diagnosis with the tissue procured, whether or not 11 portal tracts were procured.

The electronic medical records were reviewed to identify case-based details including demographics such as age and sex, indication for liver biopsy, laboratory values including platelets and international normalized ratio (INR), comorbidities, American Society of Anesthesiologists (ASA) physical status class, and presence of ascites. Prospective procedure-related data, including needle gauge and adverse events, were recorded. A single, expert gastrointestinal pathologist reviewed all samples related to the study to prospectively record the cumulative core length, number of portal tracts, maximum fragment length, number of total fragments, sample adequacy, and diagnostic yield.

\section{Procedure technique}

All subjects were sedated under monitored anesthesia care and placed in the left lateral position for the procedure. A linear echoendoscope (EG-3870UTK; Pentax Medical, Montvale, NJ, USA) was advanced through the oropharynx to the gastric body, and the left lobe of the liver was identified. The scope was positioned to optimize the endosonographic visualization so that at least $7 \mathrm{~cm}$ of the liver parenchyma could be accessed by the needle without significant intervening vasculature using Doppler imaging. A 19 G core biopsy needle (SharkCore FNB; Medtronic, Minneapolis, MN, USA) was prepared by removing the stylet and flushing the entire needle catheter with sterile saline fluid. The needle was advanced through the echoendoscope and oriented at an angle to safely pass at a maximum length into the liver parenchyma. The actuation of the needle was a swift motion into the liver parenchyma. Once actuation was achieved, the syringe was set to maximum suction at the back end of the needle. The needle was kept in position until saline was seen rising into the syringe itself, at which point suction was turned off. The needle was slowly removed, and the tissue sample was extracted with a slow saline flush directly into formalin. The majority of superfluous samples of blood were removed from the specimen using the sifting method with additional formalin fluid (Fig. 1).

Subsequently, a second biopsy was obtained from the same lobe of the liver from the transgastric position but in a different tract through the liver using the $22 \mathrm{G}$ core biopsy needle (SharkCore FNB; Medtronic). Any additional diagnostic endosonographic images required for completion of the EUS examination were obtained prior to termination of the procedure. 
Table 1. Patient Baseline Characteristics

\begin{tabular}{|c|c|c|c|c|c|}
\hline Subject & Sex & Age & $\begin{array}{l}\text { ASA } \\
\text { class }\end{array}$ & Indication for liver biopsy & Histology \\
\hline Subject 1 & $\mathrm{M}$ & 54 & 3 & Abnormal liver tests of unclear etiology & Mild steatosis without fibrosis \\
\hline Subject 2 & M & 68 & 3 & $\begin{array}{l}\text { Assess for post liver transplant acute } \\
\text { cellular rejection }\end{array}$ & No acute cellular rejection, mild fibrosis \\
\hline Subject 3 & $\mathrm{~F}$ & 48 & 2 & Abnormal liver tests of unclear etiology & $\begin{array}{l}\text { No steatosis or fibrosis } \\
\text { Intrahepatocyte iron with Kupffer cells }\end{array}$ \\
\hline Subject 4 & M & 54 & 3 & Needed staging of fibrosis & PSC with steatohepatitis and fibrosis \\
\hline Subject 5 & $\mathrm{~F}$ & 74 & 3 & Needed staging of fibrosis & Portal and lobular inflammation, no fibrosis \\
\hline Subject 6 & $\mathrm{~F}$ & 73 & 2 & Abnormal liver imaging & Large duct obstruction, stage $1-2$ fibrosis \\
\hline Subject 7 & $\mathrm{~F}$ & 55 & 3 & Needed staging of fibrosis & Mild steatosis without fibrosis \\
\hline Subject 8 & $\mathrm{~F}$ & 40 & 3 & Needed staging of fibrosis & Benign liver parenchyma without fibrosis \\
\hline Subject 9 & $\mathrm{~F}$ & 59 & 3 & Needed staging of fibrosis & Mild steatosis, Stage 1 fibrosis \\
\hline Subject 10 & $\mathrm{~F}$ & 53 & 3 & Needed staging of fibrosis & Mild macrovesicular steatosis without fibrosis \\
\hline Subject 11 & $\mathrm{~F}$ & 55 & 3 & Abnormal liver imaging & Minimal steatosis, no fibrosis \\
\hline Subject 12 & M & 45 & 2 & Needed staging of fibrosis & Benign liver parenchyma with mild steatosis, no fibrosis \\
\hline Subject 13 & $\mathrm{~F}$ & 67 & 2 & Abnormal liver imaging & Mild steatosis without fibrosis \\
\hline Subject 14 & $\mathrm{~F}$ & 40 & 2 & Abnormal liver imaging & Mild steatosis without fibrosis \\
\hline Subject 15 & $\mathrm{~F}$ & 57 & 2 & Needed staging of fibrosis & Benign liver parenchyma, no fibrosis \\
\hline Subject 16 & $\mathrm{~F}$ & 76 & 3 & Needed staging of fibrosis & $\begin{array}{l}\text { Moderate to severe bile duct inflammation, consistent with } \\
\text { PBC }\end{array}$ \\
\hline Subject 17 & $\mathrm{~F}$ & 54 & 3 & Needed staging of fibrosis & Moderate steatosis without fibrosis \\
\hline Subject 18 & $\mathrm{~F}$ & 58 & 2 & Needed staging of fibrosis & $\begin{array}{l}\text { Chronic hepatitis, plasma cell infiltration, consistent with } \\
\text { autoimmune hepatitis, without fibrosis }\end{array}$ \\
\hline Subject 19 & M & 55 & 3 & Needed staging of fibrosis & Mild to moderate steatohepatitis, with cirrhosis \\
\hline Subject 20 & $\mathrm{~F}$ & 64 & 3 & Needed staging of fibrosis & $\begin{array}{l}\text { Chronic portal and lobular inflammation, stage } 2 \text { fibrosis, } \\
\text { suggestive of drug induced liver injury or venous outflow } \\
\text { obstruction }\end{array}$ \\
\hline
\end{tabular}

ASA, American Society of Anesthesiologists; PBC, primary biliary cholangitis; PSC, primary sclerosing cholangitis.

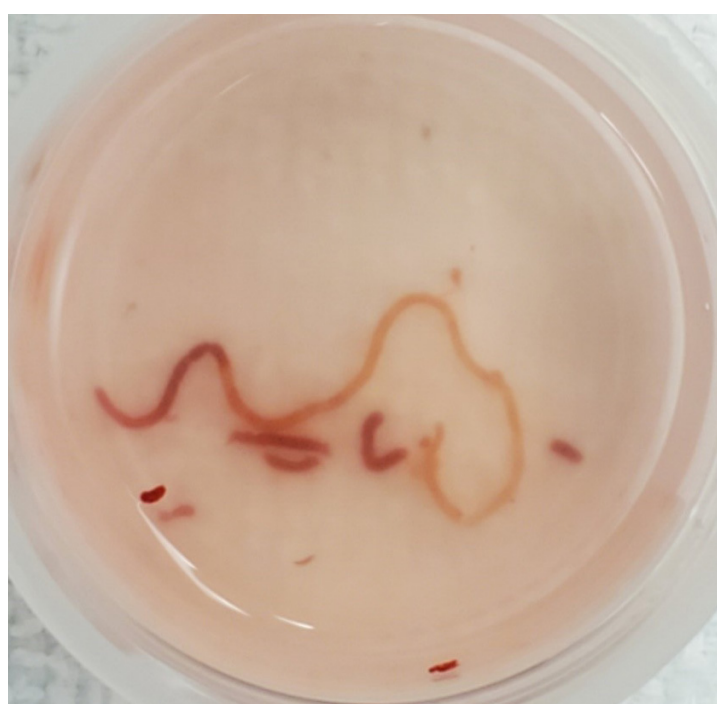

Fig. 1. Endoscopic ultrasound-guided liver biopsy core sampling, 19 gauge fine-needle aspiration needle.

\section{Statistical analysis}

Summary statistics for the two groups were calculated for specimen adequacy, cumulative length, cumulative number of tracts, number of fragments, maximum fragment length, and whether specimen was diagnostic. The Wilcoxon signedrank test was used for analysis of continuous data and McNemar's test was used for the analysis of categorical variables to determine the difference between $19 \mathrm{G}$ and $22 \mathrm{G}$ needle biopsies for EUS-LB. All statistical tests were 2-tailed at a level of significance of .05. Statistical analyses were performed using Stata software version 16 (StataCorp LLC, College Station, TX, USA). 


\section{RESULTS}

A total of 20 patients were prospectively included in this study. Fifteen $(75 \%)$ of the subjects were women. The mean age of patients in this study was 57.4 years. The median preprocedural platelet and INR values were 260 (interquartile range [IQR], 80) and 1.0 (IQR, 0), respectively. The median ASA class for patients was 3 .

The overall results are shared in Table 2. The mean cumulative core length was significantly longer for the $19 \mathrm{G}$ core sample vs. the $22 \mathrm{G}$ core sample: $2.52 \mathrm{~cm}$ and $1.27 \mathrm{~cm}$ respectively $(p<0.0001)$. The $19 \mathrm{G}$ needle also resulted in a significantly higher number of complete portal tracts (5.8 vs. 1.7, $p<0.0001$ ), and total number of tracts ( 8.8 vs. $3, p<0.0001$ ) when compared to the $22 \mathrm{G}$ needle. While there was no statistical difference between the number of fragments (11.55 vs. $9.6, p=0.179)$, the $19 \mathrm{G}$ needle yielded longer, intact fragments $(0.75 \mathrm{~cm}$ vs. $0.32 \mathrm{~cm}, p<0.0006)$. The $19 \mathrm{G}$ needle was also superior in providing an adequate and diagnostic pathologic sample. A total of $17 / 20(85 \%)$ of $19 \mathrm{G}$ samples met the criteria for diagnostic specimens vs. $2 / 20(10 \%)$ of the $22 \mathrm{G}$ samples $(p<0.0001)$. A total of $12 / 20(60 \%)$ of $19 \mathrm{G}$ samples were adequate pathologic specimens vs. $1 / 20(5 \%)$ of the $22 \mathrm{G}$ samples $(p<0.001)$. There were no adverse events in either group.

\section{DISCUSSION}

Liver biopsies are the gold standard for diagnosing and staging parenchymal liver diseases, including, but not limited to, hepatitis $C$, autoimmune hepatitis, and nonalcoholic steatohepatitis. Traditional methods of liver biopsy, including percu- taneous and transjugular biopsy, are effective, but not without limitations.

While percutaneous liver biopsy remains the most common alternative form of obtaining liver tissue, given the limited window of access to only the right lobe of the liver as well as a blind biopsy technique, percutaneous liver biopsy can miss and underestimate fibrosis staging. ${ }^{11}$ Post procedural adverse event incidence has been quoted as high as $60 \%$ within two hours of the procedure. ${ }^{4,9,12}$ The limitations of percutaneous liver biopsies include pain, an uncooperative patient, inability to perform in cases of suspected hemangiomas or echinococcal cysts, and relative contraindications in those who have a higher risk of bleeding and large body habitus. ${ }^{9}$ Consequently, many providers choose not to perform percutaneous liver biopsy for fear of complications such as severe post-procedure pain, bile peritonitis, puncturing nearby organs, and intraperitoneal hemorrhage. ${ }^{13}$ Although transjugular liver biopsies can be safely performed in patients in whom percutaneous liver biopsy is contraindicated, the reported adverse event incidence range is between $1.3 \%$ and $20.2 \%$, which includes neck hematoma and arteriovenous fistula formation., ${ }^{4,12}$

EUS-LB has been shown to have fewer complications than percutaneous or transjugular liver biopsies, while procuring a comparable amount of tissue acquisition. ${ }^{14}$ In one comparative study, percutaneous and transjugular biopsies resulted in complications of pneumothorax, significant bleeding with hepatic artery pseudoaneurysm, and multiple cases of excruciating abdominal pain while EUS-LB had no adverse events. Diagnostic yields of percutaneous and transjugular liver biopsies are between $85 \%-99 \%$ and $80 \%-97 \%$ respectively. ${ }^{13}$ EUS-LB also has a similar yield of $>90 \%$ with at least two needle passes. ${ }^{5,13,15,16}$ Additional benefits of EUS guided biopsy includes

Table 2. The Differences in Biopsy Characteristics between the $19 \mathrm{G}$ and $22 \mathrm{G}$ Needles

\begin{tabular}{lccc}
\hline Characteristics of biopsy & 19 G needle $(\boldsymbol{n}=\mathbf{2 0})$ & 22 G needle $(\boldsymbol{n}=\mathbf{2 0})$ & $\boldsymbol{p}$-value \\
\hline Cumulative length, cm & 2.52 & 1.27 & $<0.0001$ \\
Number of complete portal tracts & 5.8 & 1.7 & $<0.0001$ \\
Total number of tracts & 8.8 & 3 & $<0.0001$ \\
Number of fragments & 11.55 & 9.6 & 0.1791 \\
Maximum fragment length, cm & 0.75 & 0.32 & 0.0006 \\
Diagnostic specimen? & $3(15 \%)$ & $18(80 \%)$ & 0.0001 \\
No & $17(85 \%)$ & $2(10 \%)$ & \\
Yes & $8(40 \%)$ & $19(95 \%)$ & 0.0010 \\
Specimen adequacy? & $12(60 \%)$ & $1(5 \%)$ & 0 \\
No & 0 & 0 & \\
Yes & & & \\
Adverse events (including early and late) & & \\
\hline
\end{tabular}


high resolution imaging, ability to sample both lobes, visualization of vessels and bile ducts to prevent injury, staging and confirmation of malignancy, and ability to perform concurrent luminal upper gastrointestinal examination and intervention. ${ }^{1}$

Early data on EUS-LB are encouraging enough to presume that it will be a useful technique for the management of patients with liver disease. Further studies will be required with regard to where it falls into the clinical algorithm for various tissue acquisition techniques. At present, it will be prudent for endoscopists to study the best methods for optimizing EUSLB in terms of adequacy and safety. The primary advantage of EUS is that it may drive safer outcomes compared to other liver biopsy techniques in real-time liver parenchyma imaging during needle puncture. To further this benefit, it is necessary to reduce the number of necessary needle passes and the size of the needle gauge under direct visualization while still maintaining specimen adequacy.

Multiple studies have reported adequate EUS-LB specimens with various needle types and gauges, including $19 \mathrm{G}$ and 22 $\mathrm{G}$, but required a significant number of needle actuations in the liver, ranging from 9-20.-9,13 One study showing very high specimen adequacy with only two total needle actuations was the single-pass, single-actuation, wet suction technique by $\mathrm{Ni}$ eto et al. . This study used a $19 \mathrm{G}$ needle to obtain a specimen from the left and right lobes. Our study aimed to determine whether reducing the number of needle passes to a single actuation in the left lobe with various needle gauges, including both $19 \mathrm{G}$ and $22 \mathrm{G}$, would still be adequate. From the small sample size in this study, it is clear that a $19 \mathrm{G}$ needle provides more portal tracts and cumulative specimen length, which translate into higher specimen adequacy and diagnostic yield as compared to a $22 \mathrm{G}$ needle. Given that we only performed one single actuation with each needle, the diagnostic yield reached $85 \%$ for $19 \mathrm{G}$, and $10 \%$ for the $22 \mathrm{G}$ needle, while specimen adequacy was not optimal and only approached $60 \%$ for the $19 \mathrm{G}$ needle and $10 \%$ for the $22 \mathrm{G}$ needle. There were more diagnostic samples than adequate samples likely because in some cases the pathologist was able to make a diagnosis despite having fewer than 11 portal tracts in the tissue specimen. If we had performed 2 passes per needle, one into each lobe of the liver, and we assumed that the meaningful pathologic outcomes would double for each needle, then the $19 \mathrm{G}$ needle would likely approach $100 \%$ adequacy and diagnostic yield while still remaining suboptimal to the $22 \mathrm{G}$ needle. Thus, our conclusion is that for this specific needle design (SharkCore FNB; Medtronic), a single-pass, single-actuation, wet suction technique, with two passes of a $19 \mathrm{G}$ needle is likely the minimum number of passes and needle gauge that would result in a consistently adequate specimen. Gaining consistent specimen adequacy with a $22 \mathrm{G}$ needle would require multiple more passes/actuations. Other comparative studies have shown similar results in terms of higher diagnostic yield and specimen quality with a $19 \mathrm{G}$ compared to a $22 \mathrm{G}$ needle. ${ }^{11,12}$ That being said, other commercially available needles with different tip designs, including a tri-pronged beveled edge, may improve the quality of tissue acquisition and further reduce the number of passes required to obtain consistently adequate specimens.

The limitations of this study include the small sample size from a single center. In addition, there was likely a learning curve in the sampling technique that may have led to improved specimens as experience was gained. Despite these limitations, the difference in adequacy and yield between the two needle types was marked, and supports using a $19 \mathrm{G}$ needle for this specific tissue acquisition technique. Larger prospective studies are needed to corroborate these findings and conclusions.

\section{Conflicts of Interest}

Prashant Kedia is a consultant Boston Scientific, Medtronic, and Olympus. The authors have no potential conflicts of interest.

Funding

None.

\section{Author Contributions}

Conceptualization: Rucha M. Shah, Prashant Kedia

Data curation: RMS, Jason Schmidt, Elizabeth John, Sheila Rastegari, PK Formal analysis: RMS, EJ, SR, Priyanka Acharya, PK

Investigation: JS, PK

Methodology: PK

Project administration: PK

Resources: PA, PK

Software: PA, PK

Supervision: $\mathrm{PK}$

Validation: PA, PK

Visualization: PK

Writing-original draft: RMS, JS, EJ, SR, PK

Writing-review\&editing: RMS, JS, EJ, SR, PK

\section{ORCID}

Rucha M. Shah:

Jason Schmidt:

Elizabeth John:

Sheila Rastegari:

Priyanka Acharya:

Prashant Kedia: https://orcid.org/0000-0001-8538-8021 https://orcid.org/0000-0001-5470-7915 https://orcid.org/0000-0002-1557-386X https://orcid.org/0000-0001-8847-9930 https://orcid.org/0000-0001-6472-0902 https://orcid.org/0000-0003-3867-4492

\section{REFERENCES}

1. Schulman AR, Thompson CC, Odze R, Chan WW, Ryou M. Optimizing EUS-guided liver biopsy sampling: comprehensive assessment of needle types and tissue acquisition techniques. Gastrointest Endosc 
2017;85:419-426.

2. Pineda JJ, Diehl DL, Miao CL, et al. EUS-guided liver biopsy provides diagnostic samples comparable with those via the percutaneous or transjugular route. Gastrointest Endosc 2016;83:360-365.

3. Diehl DL, Johal AS, Khara HS, et al. Endoscopic ultrasound-guided liver biopsy: a multicenter experience. Endosc Int Open 2015;3:E210-E215.

4. Parekh PJ, Majithia R, Diehl DL, Baron TH. Endoscopic ultrasound-guided liver biopsy. Endosc Ultrasound 2015;4:85-91.

5. Mohan BP, Shakhatreh M, Garg R, Ponnada S, Adler DG. Efficacy and safety of EUS-guided liver biopsy: a systematic review and meta-analysis. Gastrointest Endosc 2019;89:238-246.e3.

6. Bazerbachi F, Vargas EJ, Matar R, et al. EUS-guided core liver biopsy sampling using a 22-gauge fork-tip needle: a prospective blinded trial for histologic and lipidomic evaluation in nonalcoholic fatty liver disease. Gastrointest Endosc 2019;90:926-932.

7. Hasan MK, Kadkhodayan K, Idrisov E, et al. Endoscopic ultrasound-guided liver biopsy using a 22-G fine-needle biopsy needle: a prospective study. Endoscopy 2019;51:818-824.

8. Ching-Companioni RA, Diehl DL, Johal AS, Confer BD, Khara HS. 19G aspiration needle versus 19G core biopsy needle for endoscopic ultrasound-guided liver biopsy: a prospective randomized trial. Endoscopy 2019;51:1059-1065.

9. Nieto J, Khaleel H, Challita Y, et al. EUS-guided fine-needle core liver biopsy sampling using a novel 19-gauge needle with modified 1-pass, 1 actuation wet suction technique. Gastrointest Endosc 2018;87:469-475.

10. Rockey DC, Caldwell SH, Goodman ZD, Nelson RC, Smith AD. Liver biopsy. Hepatology 2009;49:1017-1044.

11. Caldwell S. Liver biopsy: the reports of its demise are greatly exaggerated. Clin Transl Gastroenterol 2016;7:e171.

12. Bravo AA, Sheth SG, Chopra S. Liver biopsy. N Engl J Med 2001;344:495-500.

13. Stavropoulos SN, Im GY, Jlayer Z, et al. High yield of same-session EUS-guided liver biopsy by 19-gauge FNA needle in patients undergoing EUS to exclude biliary obstruction. Gastrointest Endosc 2012;75:310-318.

14. Shuja A, Alkhasawneh A, Fialho A, et al. Comparison of EUS-guided versus percutaneous and transjugular approaches for the performance of liver biopsies. Dig Liver Dis 2019;51:826-830.

15. Mok SR, Diehl DL, Johal AS, et al. 19 versus 22 gauge fine-needle biopsy for endoscopic ultrasound guided liver biopsy (EUS-LB): a prospective randomized trial. Gastrointest Endosc 2017;85(5 Suppl):AB473-AB474.

16. Bhat I, Malvar G, Calmet F, et al. EUS-guided liver biopsy using $22 \mathrm{G}$ FNB vs. 19G FNB needles: a prospective comparison of core specimen size and diagnostic adequacy. Gastrointest Endosc 2019;89(6 Suppl):AB293 